\title{
Behavior of Various Scalar Field Potentials under Tracking Parameters of Quintessence Class of Scalar Field Models ${ }^{\dagger}$
}

\author{
Tanisha Joshi
}

check for updates

Citation: Joshi, T. Behavior of Various Scalar Field Potentials under Tracking Parameters of Quintessence Class of Scalar Field Models. Phys. Sci. Forum 2021, 2, 39. https:// doi.org/10.3390/ECU2021-09299

Academic Editor: Peter Dunsby

Published: 22 February 2021

Publisher's Note: MDPI stays neutral with regard to jurisdictional claims in published maps and institutional affiliations.

Copyright: (C) 2021 by the author. Licensee MDPI, Basel, Switzerland. This article is an open access article distributed under the terms and conditions of the Creative Commons Attribution (CC BY) license (https:/ / creativecommons.org/licenses/by/ $4.0 /)$.
Department of Physics, Lovely Professional University, Punjab 144411, India; tanishajoshi@outlook.com + Presented at the 1st Electronic Conference on Universe, 22-28 February 2021; Available online: https://ecu2021.sciforum.net/.

\begin{abstract}
Scalar field models are known for their dynamic nature, i.e., dynamic equations of state parameters to explain the late-time cosmic acceleration of the universe. The quintessence canonical scalar field with a potential is one such field which has been introduced to account for the late time acceleration of the universe. In the present work, different kinds of scalar field potentials (under the quintessence cosmological model) are mathematically investigated using tracking parameters to examine whether these potentials show thawing or tracking behavior. Tracking parameters are considered because during cosmic evolution the dynamics of tracking depend on the variation of this particular parameter. Each potential is analyzed using this parameter and accordingly the behavior is shown. It is found that among the four potentials used, three show tracking properties and only one is shows the thawing property as per the tracking parameter. The tracking and thawing properties/behavior are discussed in the Results and Discussion sections of the paper.
\end{abstract}

Keywords: tracker behavior; scalar fields; quintessence; thawing behavior; cosmology

\section{Introduction}

The accelerating expansion of the universe predicted by the observations of type Ia supernovae, published by the High-Z Supernova Search Team in 1998 [1] and the Supernova Cosmology Project in 1999 [2] suggested the concept of dark energy. Dark energy is a hypothetical form of energy pervading the whole of space. It is responsible for the accelerated expansion of the universe as it has strong negative pressure leading to gravitational repulsion (repulsive force). To understand this dark energy concept, various candidates have been put forward. These include the cosmological constant (leading to the coincidence problem) [3], quantum field theory (leading to the $\lambda$ problem) [4], scalar field models and brane world models, etc. Though there are many candidates (and many theories) of dark energy, none of them are captivating enough to decode the mystery of dark energy.

Scalar fields come to light through particle physics and string theory. Regarded as one of the candidates for dark energy, there are a wide variety of scalar field dark energy models (quintessence, tachyonic, k-essence, etc.). The simplest field mimicking dark energy is a canonical scalar field with a potential known as quintessence [5-7]. The main difference between quintessence and the cosmological constant is that quintessence is dynamic in nature. This field is capable of reproducing the late-time accelerated behavior. It has an equation of state given by $w_{\varphi}=\frac{p_{\varphi}}{\rho_{\varphi}}$, where is $p_{\varphi}$ pressure, $\rho_{\varphi}$ is energy density. Quintessence scalar field models are broadly classified into two classes [8-10]; (I) thawing models and (II) freezing models. Thawing models are slow-roll models which depend on initial value conditions whereas freezing models are fast-roll models, insensitive to the initial conditions but over the entire range of scalar field, they depend on the shape of the potential. P. Steinhardt [11] was the first to introduce tracker fields. Tracker fields are basically a class of quintessence model consisting of a scalar field rolling down a potential. 
They were introduced to avoid the problem of initial value conditions. A tracker model consists of a tracker solution, tracker field and tracker equation (with quintessence as an equation). For detailed analysis of tracker fields see the literature [12-14]. ' $\Gamma^{\prime}$ is the tracking parameter, and the properties of $\Gamma$ determine whether the tracking solutions exist or not. For any kind of potential $\Gamma$ can be $>,<$ or $=1$.

This paper proceeds as follows: In Section 2, the mathematical background for a quintessence cosmological model is set up for a tracking parameter. In Section 3, we mathematically examine the tracking parameter with various potentials to check their tracking behavior and the results are highlighted in Table 1. In Section 4, the results are concluded with brief discussion. Finally, we conclude the paper in Section 5.

Table 1. Behavior shown by potentials used for quintessence cosmological model.

\begin{tabular}{cccc}
\hline S.No. & Potential $(V(\varphi))$ & Tracking Parameter $(\Gamma)$ & Value of ' $\boldsymbol{u}^{\prime}(\boldsymbol{u} \in \mathbf{R})$ \\
\hline 1. & $V_{0} \alpha e^{(1-\varphi)}$ & $\Gamma=1$ & 1 \\
\hline 2. & $V_{0} \alpha(1-\varphi)^{-1}$ & $\Gamma>1$ & -1 \\
\hline 3. & $V_{0} \alpha\left(\frac{\varphi}{\varphi-\varphi^{2}}\right)$ & $\Gamma>1$ & 1 \\
\hline 4. & $V_{0} \cosh \left(\alpha \varphi^{u}\right)$ & $\Gamma<1$ & $\begin{array}{c}\text { For all positive and } \\
\text { negative values of } u .\end{array}$ \\
\hline
\end{tabular}

\section{Mathematical Background}

The action proposed for quintessence [15] scalar field ' $\varphi$ ' gives the action as

$$
S=\int d^{4} x \sqrt{-g}\left[\frac{-1}{2}(\llbracket \nabla \varphi) \rrbracket^{2}-V(\varphi)\right]
$$

The energy density and pressure for a spatially homogeneous quintessence scalar field can be obtained from the Lagrangian

$$
L_{q u i n}=\frac{1}{2} \partial^{i} \varphi \partial^{i} \varphi-V(\varphi)
$$

As

$$
\begin{aligned}
\rho_{\varphi} & =\frac{1}{2} \dot{\varphi}^{2}+V(\varphi) ; \\
p_{\varphi} & =\frac{1}{2} \dot{\varphi}^{2}-V(\varphi)
\end{aligned}
$$

where, $\rho_{\varphi}$ is the energy density of scalar field, $p_{\varphi}$ is the pressure density of scalar field and $V(\varphi)$ is the scalar field potential.

Equation (1) is varied with respect to the scalar field to get the equation of motion as

$$
\ddot{\varphi}+3 H \dot{\varphi}+V^{\prime}(\varphi)=0
$$

where ' $\varphi$ ' is a function of time and $V^{\prime}(\varphi)$ is the derivative with respect to $\varphi$. This equation of motion describes how the scalar field evolves with time. Here, $H=\frac{\dot{a}}{a}$ is the Hubble parameter which denotes the expansion rate of universe.

The equation of the state parameter $\left(w_{\varphi},\right)$ of quintessence is

$$
w_{\varphi}=\frac{p_{\varphi}}{\rho_{\varphi}}=\frac{\frac{1}{2} \varphi^{2}-V(\varphi)}{\frac{1}{2} \varphi^{2}+V(\varphi)} \quad \geq 1
$$


and the density parameter is

$$
\Omega_{\varphi}=\frac{\rho_{\varphi}}{3 H^{2} M_{p l}^{2}}=\frac{\rho_{\varphi}}{\rho_{\varphi}+\rho_{m}}
$$

where, $\rho_{m}$ is matter density and $M_{p l}$ is the Planck mass.

Moreover,

$$
\dot{\rho_{m}}+3 H \rho_{m}=0
$$

Now we define the dimensionless parameters,

$$
\begin{gathered}
\lambda=\frac{-m_{p} V^{\prime \prime}}{V} \\
\Gamma=\frac{V V^{\prime \prime}}{\left(V^{\prime}\right)^{2}}
\end{gathered}
$$

which are functions of the field $\lambda=\lambda(\varphi)$ and $\Gamma=\Gamma(\varphi)$, where ' $\lambda$ ' parametrizes the slope of the potential and ' $\Gamma$ ' is called the tracking parameter, and where $V^{\prime \prime}$ and $V^{\prime}$ are differentiations with respect to the scalar field, $\varphi$, and $V$ is the scalar field potential.

The potential is shallow for small values of $\lambda$ (a tracker solution leads to small $\lambda$ ), and if the field dominates the universe, it could become quintessence.

Taking the time derivative of (9) and using (10), we obtain

$$
\frac{\dot{\lambda}}{\lambda^{2}}=\frac{-\dot{\varphi}}{m_{p}}(\Gamma-1)
$$

From Equations (5)-(8), we obtain the following equations for $w$ and $\Omega_{\varphi}$ (see [16-18])

$$
\begin{gathered}
w^{\prime}=(1-w)\left[-3(1+w)+\lambda \sqrt{3(1+w) \Omega_{\varphi}}\right] \\
\Omega_{\varphi}^{\prime}=-3 w \Omega_{\varphi}\left(1-\Omega_{\varphi}\right) \\
\lambda^{\prime}=-\sqrt{3(1+w) \Omega_{\varphi}}(\Gamma-1) \lambda^{2}
\end{gathered}
$$

where a prime represents a derivative with respect to $N=\ln a$. There are several different cases for the evolution of $w$, depending on the initial conditions and field potential.

\section{Results}

The four potentials of different forms (power law, exponential and hyperbolic form) are defined as:

(a) Exponential form: $V(\varphi)=V_{0} \alpha e^{(1-\varphi)^{u}}$

(b) Power law form: $V(\varphi)=V_{0} \alpha(1-\varphi)^{u}$ and $V(\varphi)=V_{0} \alpha\left(\frac{\varphi}{\varphi-\varphi^{2}}\right)^{u}$

(c) Hyperbolic form [19]: $V(\varphi)=V_{0} \cosh \left(\alpha \varphi^{u}\right)$

where, $V_{0}, \alpha$ and $u$ are real constants in which $\alpha$ and $u$ are dimensionless and $V_{0}$ is dimensionful. Here, first the analysis is done keeping $u \in R$, for all values in the range $R$. Then, a particular value of $u(-1$ and 1$)$ is chosen to study the behavior of these potentials. Furthermore, other values are equally appealing provided they are in a range of R. Here we consider $u=-1$ and 1 respectively, for the potentials described above to proceed further towards its mathematical analysis.

Analysis

(a) $\quad V(\varphi)=V_{0} \alpha e^{(1-\varphi)^{u}}$

$$
V^{\prime}(\varphi)=-V_{0} \alpha u(1-\varphi)^{u-1} e^{(1-\varphi)^{u}}
$$




$$
\begin{gathered}
V^{\prime \prime}(\varphi)=V_{0} \alpha u^{2}(1-\varphi)^{2 u-2} e^{(1-\varphi)^{u}}+(u-1) u(1-\varphi)^{u-2} e^{(1-\varphi)^{u}} \\
\Gamma=\frac{V V^{\prime \prime}}{\left(V^{\prime}\right)^{2}} \\
\Gamma=\frac{V_{0} \alpha e^{(1-\varphi)^{u}} V_{0} \alpha u^{2}(1-\varphi)^{2 u-2} e^{(1-\varphi)^{u}}+(u-1) u(1-\varphi)^{u-2} e^{(1-\varphi)^{u}}}{\left(-V_{0} \alpha u(1-\varphi)^{u-1} e^{\left.(1-\varphi)^{u}\right) 2}\right.} \\
\Gamma=1+\frac{\left(u^{2}-u\right)}{u^{2}(1-\varphi)^{u}}
\end{gathered}
$$

For $u=0, \Gamma=$ undefined; From (15), $u=1, \Gamma=1$

For positive and negative values of $u, \Gamma>1$.

(b) $\quad V(\varphi)=V_{0} \alpha(1-\varphi)^{u}$

$$
\begin{gathered}
V^{\prime}(\varphi)=-V_{0} \alpha u(1-\varphi)^{u-1} \\
V^{\prime \prime}(\varphi)=V_{0} \alpha u(u-1)(1-\varphi)^{u-2} \\
\Gamma=\frac{V V^{\prime \prime}}{\left(V^{\prime}\right)^{2}} \\
\Gamma=\frac{V_{0} \alpha(1-\varphi)^{u} V_{0} \alpha u(u-1)(1-\varphi)^{u-2}}{\left(-V_{0} \alpha u(1-\varphi)^{u-1}\right)^{2}} \\
\Gamma=1-\frac{1}{u}
\end{gathered}
$$

For $u=0, \Gamma$ is undefined. From (16), $u=-1, \Gamma>1$.

For negative values of $u, \Gamma>1$.

For positive values of $u, \Gamma<1$.

(c) $V(\varphi)=V_{0} \alpha\left(\frac{\varphi}{\varphi-\varphi^{2}}\right)^{u}$

$$
\begin{gathered}
V^{\prime}(\varphi)=-V_{0} \alpha u \frac{\left(\frac{\varphi}{\varphi-\varphi^{2}}\right)^{u}}{(\varphi-1)} \\
V^{\prime \prime}(\varphi)=V_{0} \alpha u(u+1) \frac{\left(\frac{\varphi}{\varphi-\varphi^{2}}\right)^{u}}{(\varphi-1)^{2}} \\
\Gamma=\frac{V V^{\prime \prime}}{\left(V^{\prime}\right)^{2}} \\
\Gamma=\frac{V_{0} \alpha u(u+1) \frac{\left(\frac{\varphi}{\varphi-\varphi^{2}}\right)^{u}}{(\varphi-1)^{2}} V_{0} \alpha\left(\frac{\varphi}{\varphi-\varphi^{2}}\right)^{u}}{\left(-V_{0} \alpha u \frac{\left(\frac{\varphi}{\varphi-\varphi^{2}}\right)^{u}}{(\varphi-1)}\right)^{2}} \\
\Gamma=1+\frac{1}{u}
\end{gathered}
$$

For $u=0, \Gamma$ is undefined. From (17), $u=1, \Gamma>1$.

For negative values of $u, \Gamma<1$.

For positive values of $\mathrm{u}, \Gamma>1$.

(d) $V(\varphi)=V_{0} \cosh \left(\alpha \varphi^{u}\right)=\frac{V_{0}}{2}\left(e^{\alpha \varphi^{u}}+e^{-\alpha \varphi^{u}}\right)$

For $u>0, V(\varphi)=\frac{V_{0}}{2} e^{\alpha \varphi^{u}}$

$$
\begin{gathered}
V^{\prime}(\varphi)=\frac{V_{0}}{2} u \alpha e^{\alpha \varphi^{u-1}} \\
V^{\prime \prime}(\varphi)=\frac{V_{0}}{2} u \alpha^{2}(u-1) e^{\alpha \varphi^{u-2}}
\end{gathered}
$$




$$
\begin{gathered}
\Gamma=\frac{V V^{\prime \prime}}{\left(V^{\prime}\right)^{2}} \\
\Gamma=\frac{\frac{V_{0}}{2} e^{\alpha \varphi^{u}} \quad \frac{V_{0}}{2} u \alpha^{2}(u-1) e^{\alpha \varphi^{u-2}}}{\left(\frac{V_{0}}{2} u \alpha e^{\alpha \varphi^{u-1}}\right)^{2}} \\
\Gamma=1-\frac{1}{u}
\end{gathered}
$$

For $u<0, V(\varphi)=\frac{V_{0}}{2} e^{-\alpha \varphi^{u}}$

Similarly, as $u>0$,

$$
\Gamma=1-\frac{1}{u}
$$

which implies for positive or negative values of $u, \Gamma<1$.

\section{Discussion}

It can be seen from Table 1 that only three potentials show tracking behavior $(\Gamma \geq 1)$. The potentials showing tracking behavior are fast-roll (freezing) potentials. The hyperbolic potential shows thawing behavior $(\Gamma<1)$ and is called a slow-roll (thawing) potential. The following discusses their behavior.

$\Gamma \geq 1$ : The potential $V_{0} \alpha e^{(1-\varphi)}$ is a scaling attractor for $\Gamma=1$ It is a special case of tracker, known as scaling freezing. There exists an attractor solution to the evolution of the field, but the attractor is not a tracker. In this type of model, during most of the cosmological evolution, scalar field density and its equation of state remains close to that of the dominant background matter.

$\Gamma>1$ : The potential $V_{0} \alpha(1-\varphi)^{-1}$ and $V_{0} \alpha\left(\frac{\varphi}{\varphi-\varphi^{2}}\right)$ are attractors for $\Gamma>1$. (known as the tracking condition). This kind of attractor is called a tracker. From Equation (12), as $|\lambda|$ diminishes with time, $\Omega_{\varphi}$ grows, which implies that quintessence dominates the universe, as required. As time passes, the potential becomes shallower. In this case, during most of the cosmological evolution, scalar field density and its equation of state remains less than that of dominant background matter. This condition is equivalent to the case where $\left|\frac{V \prime}{V}\right|$ decreases as $\mathrm{V}$ decreases.

$\Gamma<1$ : The potential $V_{0} \cosh \left(\alpha \varphi^{u}\right)$ is a thawing potential for $\Gamma<1$. Thawing potentials does not enjoy the privilege of attractor behavior and should have initial conditions explained. At late times, the potential becomes shallow resulting in the gradual slowdown of the field. Thawing potentials (that indulge slow-roll conditions) come up naturally to produce $w$ (equation of state) near -1 , converging to a single, universal behavior.

From a future work perspective, possible analytic solutions of these potentials can be done for the dark energy equation of state, expressed in terms of several free parameters and using cosmological observations one can also put cosmological constraints on these various forms of potentials to get some new insights.

\section{Conclusions}

In this work, we investigated various (power law, exponential and hyperbolic form) scalar field potentials under the quintessence cosmological model to examine whether these potentials show tracking behavior or not. We analyzed this tracking behavior using a tracking parameter for each potential and accordingly we produced results. It was found that among the four potentials used for examining tracking behavior, three (power law and exponential) show tracking properties and one (hyperbolic) shows the thawing property.

Conflicts of Interest: The author declare no conflict of interest. 


\section{References}

1. Riess, A.G.; Filippenko, A.V.; Challis, P.; Clocchiatti, A.; Diercks, A.; Garnavich, P.M.; Gilliland, R.L.; Hogan, C.J.; Jha, S.; Kirshner, R.P.; et al. Observational evidence from supernovae for an accelerating universe and a cosmological constant. Astron. J. 1998, 116, 1009. [CrossRef]

2. Perlmutter, S.; Aldering, G.; Goldhaber, G.; Knop, R.A.; Nugent, P.; Castro, P.G.; Deustua, S.; Fabbro, S.; Goobar, A.; Groom, D.E.; et al. Measurements of $\Omega$ and $\Lambda$ from 42 high-redshift supernovae. Astrophys. J. 1999, 517, 565. [CrossRef]

3. Martin, J. Everything you always wanted to know about the cosmological constant problem (but were afraid to ask). C. R. Phys. 2012, 13, 566-665. [CrossRef]

4. Zlatev, I.; Wang, L.; Steinhardt, P.J. Quintessence, cosmic coincidence, and the cosmological constant. Phys. Rev. Lett. 1999, 82, 896. [CrossRef]

5. Weinberg, S. The cosmological constant problem. Rev. Mod. Phys. 1989, 61, 1.

6. Nojiri, S.; Bamba, K.; Capozziello, S.; Odinstov, S.D. Dark energy cosmology: The equivalent description via different theoretical models and cosmography tests. Astrophys. Space Sci. 2012, 342, 155.

7. Ratra, B.; Peebles, P.J. Cosmological consequences of a rolling homogeneous scalar field. Phys. Rev. D 1988, 37, 3406. [CrossRef] [PubMed]

8. Tsujikawa, S. Quintessence: A review. Class. Quantum Gravity 2013, 30, 214003. [CrossRef]

9. Caldwell, R.R.; Linder, E.V. Limits of quintessence. Phys. Rev. Lett. 2005, 95, 141301. [CrossRef] [PubMed]

10. Chiba, T. Slow-roll thawing quintessence. Phys. Rev. D 2009, 79, 083517. [CrossRef]

11. Scherrer, R.J.; Sen, A.A. Thawing quintessence with a nearly flat potential. Phys. Rev. D 2008, 77, 083515. [CrossRef]

12. Steinhardt, P.J.; Wang, L.; Zlatev, I. Cosmological tracking solutions. Phys. Rev. D 1999, 59, 123504. [CrossRef]

13. Johri, V.B. Search for tracker potentials in quintessence theory. Class. Quantum Gravity 2002, 19, 5959. [CrossRef]

14. Johri, V.B. Constraints over cosmological constant and quintessence fields in an accelerating Universe. arXiv 2000, arXiv:astro$\mathrm{ph} / 0007079$.

15. Ford, L.H. Cosmological-constant damping by unstable scalar fields. Phys. Rev. D 1987, 35, 2339. [CrossRef] [PubMed]

16. Copeland, E.J.; Liddle, A.R.; Wands, D. Exponential potentials and cosmological scaling solutions. Phys. Rev. D 1998, 57, 4686. [CrossRef]

17. Dutta, S.; Scherrer, R.J. Hilltop quintessence. Phys. Rev. D 2008, 78, 123525. [CrossRef]

18. De la Macorra, A.; Piccinelli, G. Cosmological evolution of general scalar fields and quintessence. Phys. Rev. D 2000, 61, 123503. [CrossRef]

19. Yang, W.; Shahalam, M.; Pal, B.; Pan, S.; Wang, A. Constraints on quintessence scalar field models using cosmological observations. Phys. Rev. D 2019, 100, 023522. [CrossRef] 